\title{
VON NEUMANN COORDINATIZATION IS NOT FIRST-ORDER
}

\author{
FRIEDRICH WEHRUNG
}

\begin{abstract}
A lattice $L$ is coordinatizable, if it is isomorphic to the lattice $\boldsymbol{L}(R)$ of principal right ideals of some von Neumann regular ring $R$. This forces $L$ to be complemented modular. All known sufficient conditions for coordinatizability, due first to J. von Neumann, then to B. Jónsson, are first-order. Nevertheless, we prove that coordinatizability of lattices is not first-order, by finding a non-coordinatizable lattice $K$ with a coordinatizable countable elementary extension $L$. This solves a 1960 problem of B. Jónsson. We also prove that there is no $\mathcal{L}_{\infty, \infty}$ statement equivalent to coordinatizability. Furthermore, the class of coordinatizable lattices is not closed under countable directed unions; this solves another problem of B. Jónsson from 1962.
\end{abstract}

\section{INTRODUCTION}

A coordinatization theorem is a statement that expresses a class of geometric objects in algebraic terms. Hence it is a path from synthetic geometry to analytic geometry. While the former includes lattice theory, as, for example, abundantly illustrated in M.K. Bennett's survey paper [2], the latter is more often written in the language of rings and modules. Nevertheless the concepts of analytic and synthetic geometry will not let themselves be captured so easily. For example, the main result of E. Hrushovski and B. Zilber [13, Theorem A] may certainly be viewed as a coordinatization theorem, with geometric objects of topological nature.

It should be no surprise that coordinatization theorems are usually very difficult results. The classical coordinatization theorem of Arguesian affine planes (as, for example, presented in E. Artin [1, Chapitre II]) was extended over the last century to a huge work on modular lattices, which also brought surprising and deep connections with coordinatization results in universal algebra, see the survey paper by C. Herrmann [1]. We cite the following milestone, due to J. von Neumann [24].

Von Neumann's Coordinatization Theorem. If a complemented modular lattice $L$ has a spanning finite homogeneous sequence with at least four elements, then $L$ is coordinatizable, that is, there exists a von Neumann regular ring $R$ such that $L$ is isomorphic to the lattice $\boldsymbol{L}(R)$ of all principal right ideals of $R$.

We refer the reader to Sections 24 for precise definitions. We observe that while the statement that a lattice is coordinatizable is, apparently, "complicated" (it begins with an existential quantifier over regular rings), von Neumann's sufficient condition is logically simple - for example, having a spanning homogeneous sequence with four elements is a first-order condition.

Date: November 8, 2018.

2000 Mathematics Subject Classification. 06C20, 06C05, 03C20, 16E50.

Key words and phrases. Lattice; complemented; modular; 2-distributive; coordinatizable; ring; von Neumann regular; center. 
The strongest known extension of von Neumann's Coordinatization Theorem is due to B. Jónsson [15]. For further coordinatization results of modular lattices, see, for example, B. Jónsson and G. Monk [18, A. Day and D. Pickering [6], or the survey by M. Greferath and S. E. Schmidt [10].

Jónsson's Extended Coordinatization Theorem. Every complemented Arguesian lattice L with a "large partial 3-frame" is coordinatizable.

Although having a large partial 3-frame is, apparently, not a first-order condition, we prove in Section [10 by using the dimension monoid introduced in F. Wehrung [27, that it can be expressed by a single first-order sentence.

Is coordinatizability first-order? The question was raised by B. Jónsson in the Introduction of [15]. We quote the corresponding excerpt.

A complete solution to our problem would consist in an axiomatic characterization of the class of all coordinatizable lattices. However, this seems to be an extremely difficult problem, and in fact it is doubtful that any reasonable axiom system can be found.

In the present paper, we confirm Jónsson's negative guess, in particular removing the word "reasonable" from the second sentence above. In fact, our negative solution even holds for a restricted class of complemented modular lattices, namely, those that satisfy the identity of 2-distributivity,

$$
x \vee\left(y_{0} \wedge y_{1} \wedge y_{2}\right)=\left(x \vee\left(y_{0} \wedge y_{1}\right)\right) \wedge\left(x \vee\left(y_{0} \wedge y_{2}\right)\right) \vee\left(x \vee\left(y_{1} \wedge y_{2}\right)\right)
$$

A few examples of 2-distributive modular lattices are diagrammed on Figure 1. The subspace lattice of a three-dimensional vector space is not 2-distributive. An important characterization of 2-distributivity for modular lattices is provided by $\mathrm{C}$. Herrmann, D. Pickering, and M. Roddy [12]: A modular lattice is 2-distributive iff it can be embedded into the subspace lattice of a vector space over any field. So, in some sense, the theory of 2-distributive modular lattices is the "characteristic-free" part of the theory of modular lattices.
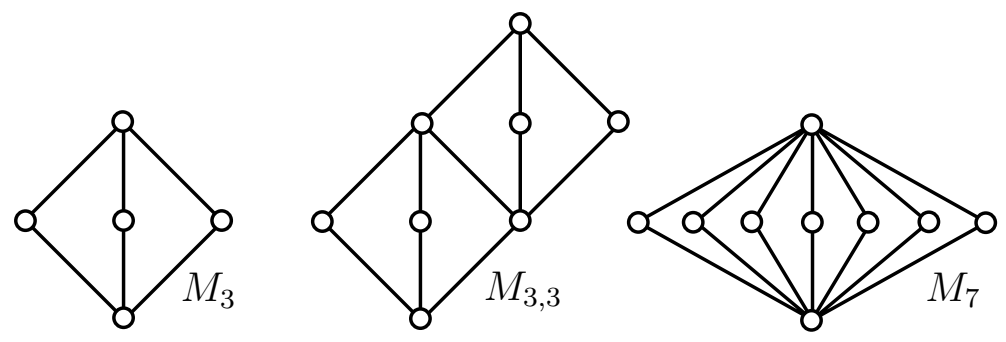

FiguRE 1. A few 2-distributive modular lattices.

We also find a large proper class of non-coordinatizable lattices with spanning $M_{\omega}$, see Theorem 9.3 This result is sufficient to ensure that there is no $\mathcal{L}_{\infty, \infty}$ statement equivalent to coordinatizability (see Theorem 9.4).

We put $\mathbb{N}=\omega \backslash\{0\}$, and we denote by $\mathbb{P}$ the set of all prime numbers. For a prime $p$, we denote by $\mathbb{F}_{p^{\infty}}$ an (the) algebraic closure of the prime field $\mathbb{F}_{p}$ with $p$ elements, and we put $\mathbb{F}_{q}=\left\{x \in \mathbb{F}_{p^{\infty}} \mid x^{q}=x\right\}$, for any power $q$ of $p$. Hence $\mathbb{F}_{q}$ is a (the) field with $q$ elements, and $\mathbb{F}_{m}$ is a subfield of $\mathbb{F}_{n}$ iff $n$ is a power of $m$. 
Following standard set-theoretical notation, we denote by $\omega$ the chain of all natural numbers and by $\omega_{1}$ the first uncountable ordinal.

If $\alpha$ is an equivalence relation on a set $X$, we denote by $[x]_{\alpha}$ the $\alpha$-equivalence class of $x$ modulo $\alpha$, for every $x \in X$. If $f: X \rightarrow Y$ is a map, we put $f[Z]=$ $\{f(x) \mid x \in Z\}$, for any $Z \subseteq X$. For an infinite set $I$, a family $x=\left\langle x_{i} \mid i \in I\right\rangle$ is almost constant, if there exists a (necessarily unique) $a$ such that $\left\{i \in I \mid x_{i} \neq a\right\}$ is finite, and then we put $a=x(\infty)$, the limit of $x$.

\section{LATTICES}

Standard textbooks on lattice theory are G. Birkhoff [3, G. Grätzer 9], and R. N. McKenzie, G. F. McNulty, and W.F. Taylor [19]. We say that a lattice $L$ is bounded, if it has a zero (i.e., a least element), generally denoted by 0 , and a unit (i.e., a largest element), generally denoted by 1 . For an element $a$ in a lattice $L$, we put

$$
L \uparrow a=\{x \in L \mid x \leq a\} \text {, the principal ideal generated by } a .
$$

We say that $L$ is modular, if it satisfies the identity

$$
x \wedge(y \vee(x \wedge z))=(x \wedge y) \vee(x \wedge z) .
$$

We shall sometimes mention a stronger identity than modularity, the so-called Arguesian identity, which can be found, for example, in [9 Section IV.4]. The Arguesian identity holds in every lattice of permuting equivalence relations (see B. Jónsson [14] or 9, Section IV.4]). In particular, it holds in the lattice Sub $M$ of all submodules of any right module $M$ over any ring.

In case $L$ has a zero and for $a, b, c \in L$, we let $c=a \oplus b$ hold, if $c=a \vee b$ and $a \wedge b=0$. It is well-known that the partial operation $\oplus$ is associative in case $L$ is modular (see [20, Satz I.1.8] or [27, Proposition 8.1]).

We say that $L$ is complemented, if it is bounded and every $x \in L$ has a complement, that is, an element $y \in L$ such that $x \oplus y=1$. We say that $L$ is sectionally complemented, if $L$ has a zero and every principal ideal of $L$ is a complemented sublattice.

In case $L$ has a zero, the relations of perspectivity, $\sim$, and subperspectivity, $\lesssim$, are defined in $L$ by

$$
\begin{aligned}
& x \sim y, \text { if } \exists z \in L \text { such that } x \oplus z=y \oplus z, \\
& x \lesssim y, \text { if } \exists z \in L \text { such that } x \oplus z \leq y \oplus z .
\end{aligned}
$$

In case $L$ is sectionally complemented and modular, $x \lesssim y$ iff there exists $x^{\prime} \leq y$ (resp., $y^{\prime} \geq x$ ) such that $x \sim x^{\prime}$ (resp., $y \sim y^{\prime}$ ) (see [24, Theorem I.3.1]).

An element $a$ in a lattice $L$ is neutral (see [9, Section III.3]), if $\{a, x, y\}$ generates a distributive sublattice of $L$, for all $x, y \in L$. An ideal $I$ of a lattice $L$ is neutral (see [9, Section III.4]), if $I$ is a neutral element of the lattice Id $L$ of ideals of $L$. Then $I$ is a distributive ideal of $L$, that is, the equivalence relation $\equiv_{I}$ of $L$ defined by

$$
x \equiv_{I} y \Longleftrightarrow \exists u \in I \text { such that } x \vee u=y \vee u \text {, for all } x, y \in L,
$$

is a congruence of $L$. Then we denote by $L / I$ the quotient lattice $L / \equiv_{I}$, and we put $[x]_{I}=[x]_{\equiv_{I}}$, the $\equiv_{I}$-equivalence class of $x$, for any $x \in L$.

In case $L$ is sectionally complemented, this can be easily expressed in terms of the relations of perspectivity and subperspectivity in $L$. The following result is proved in [3, Theorem III.13.20]. 
Proposition 2.1. Let I be an ideal of a sectionally complemented modular lattice $L$. Then $I$ is neutral iff $x \sim y$ and $y \in I$ implies that $x \in I$, for all $x, y \in L$.

Corollary 2.2. Let $L$ be a sectionally complemented modular lattice. An element $u \in L$ is neutral iff $x \lesssim u$ and $x \wedge u=0$ implies that $x=0$, for all $x \in L$.

For a positive integer $n$, a homogeneous sequence of order $n$ in a lattice $L$ with zero is an independent (see [9] Definition IV.1.9]) sequence $\left\langle a_{0}, \ldots, a_{n-1}\right\rangle$ of pairwise perspective elements of $L$.

The center of a bounded lattice $L$, denoted by cen $L$, is the set of all complemented neutral elements of $L$. The elements of cen $L$ correspond exactly to the direct decompositions of $L$. This can be expressed conveniently in the following way (see [9, Theorem III.4.1]).

Lemma 2.3. Let $L$ be a bounded lattice and let $a, b \in L$. Then the following are equivalent:

(i) There are bounded lattices $A$ and $B$ and an isomorphism $f: L \rightarrow A \times B$ such that $f(a)=\langle 1,0\rangle$ and $f(b)=\langle 0,1\rangle$.

(ii) The pair $\langle a, b\rangle$ is complementary in cen $L$, that is, $a, b \in \operatorname{cen} L$ and $a \oplus b=1$.

Furthermore, $\operatorname{cen}(L\lceil a)=(\operatorname{cen} L)\lceil a$, for any $a \in \operatorname{cen} L$.

For the following result we refer the reader to [9, Theorem III.2.9].

Proposition 2.4. The center of a bounded lattice $L$ is a Boolean sublattice of $L$.

\section{REgular Rings}

All our rings will be associative. Most of the time they will also be unital, with a few exceptions. A ring $R$ is (von Neumann) regular, if every element $a$ of $R$ has a quasi-inverse, that is, an element $b$ of $R$ such that $a b a=a$. For a regular ring $R$, the set $\boldsymbol{L}(R)$ of principal right ideals of $R$, that is,

$$
\boldsymbol{L}(R)=\{x R \mid x \in R\}=\left\{x R \mid x \in R, x^{2}=x\right\}
$$

partially ordered by inclusion, is a sectionally complemented modular lattice (see Section 21), with least element $\left\{0_{R}\right\}$.

Hence every coordinatizable lattice is sectionally complemented and modular. It is observed in B. Jónsson [17. Section 9] that a bounded lattice is coordinatizable iff it can be coordinatized by a regular, unital ring.

We shall need the following classical result (see K. R. Goodearl [8, Theorem 1.7], or K. D. Fryer and I. Halperin [7, Section 3.6] for the general, non-unital case).

Proposition 3.1. For any regular ring $R$ and any positive integer $n$, the ring $\mathrm{M}_{n}(R)$ of all $n \times n$ matrices with entries in $R$ is regular.

We shall need a more precise form of the result stating that $\boldsymbol{L}(R)$ is a lattice, proved in [7. Section 3.2].

Proposition 3.2. Let $R$ be a regular ring and let $a, b \in R$ with $a^{2}=a$. Furthermore, let $u$ be a quasi-inverse of $b-a b$. Then the following statements hold:

(i) Put $c=(b-a b) u$. Then $a R+b R=(a+c) R$.

(ii) Suppose that $b^{2}=b$ and put $d=u(b-a b)$. Then $a R \cap b R=(b-b d) R$. 
A ring $R$ endowed with its canonical structure of right $R$-module will be denoted by $R_{R}$.

Corollary 3.3. Let $R$ be a regular ring. Then $\boldsymbol{L}(R)$ is a sectionally complemented sublattice of $\operatorname{Sub}\left(R_{R}\right)$.

Remember that $\operatorname{Sub}\left(R_{R}\right)$ is an Arguesian lattice; hence so is $\boldsymbol{L}(R)$.

We shall also use the following easy consequence of Proposition [3.2 already observed in F. Micol's thesis 21.

Corollary 3.4. Let $R$ and $S$ be regular rings and let $f: R \rightarrow S$ be a ring homomorphism. Put $I=\operatorname{ker} f$. Then the following statements hold:

(i) There exists a unique map $g: \boldsymbol{L}(R) \rightarrow \boldsymbol{L}(S)$ such that $g(x R)=f(x) S$ for all $x \in R$. We shall denote this map by $\boldsymbol{L}(f)$.

(ii) $\boldsymbol{L}(f)$ is a 0-lattice homomorphism from $\boldsymbol{L}(R)$ to $\boldsymbol{L}(S)$.

(iii) There is an isomorphism $\varepsilon$ from $\operatorname{ker} \boldsymbol{L}(f)$ onto $\boldsymbol{L}(I)$, defined by the rule $\varepsilon(x R)=x I$, for all $x \in I$.

(iv) If $f$ is a ring embedding, then $\boldsymbol{L}(f)$ is a lattice embedding.

(v) If $f$ is surjective, then $\boldsymbol{L}(f)$ is surjective.

Furthermore, the correspondence $R \mapsto \boldsymbol{L}(R), f \mapsto \boldsymbol{L}(f)$ defines a functor from the category of regular rings and ring homomorphisms to the category of sectionally complemented modular lattices and 0-lattice homomorphisms. This functor preserves direct limits.

In particular, if we identify $\{x R \mid x \in I\}$ with $\boldsymbol{L}(I)$ (via the isomorphism $\varepsilon$ ), then we obtain the isomorphism $\boldsymbol{L}(R / I) \cong \boldsymbol{L}(R) / \boldsymbol{L}(I)$.

The following result sums up a few easy preservation statements.

\section{Proposition 3.5.}

(i) Any neutral ideal of a coordinatizable lattice is coordinatizable.

(ii) Any homomorphic image of a coordinatizable lattice is coordinatizable.

(iii) Any reduced product of coordinatizable lattices is coordinatizable.

Proof. (i) Let $R$ be a regular ring and let $\boldsymbol{I}$ be a neutral ideal of $\boldsymbol{L}(R)$. The subset $I=\{x \in R \mid x R \in \boldsymbol{I}\}$ is a two-sided ideal of $R$ (see [26. Theorem 4.3]), thus, in particular, it is a regular ring in its own right (see [8, Lemma 1.3]). Furthermore, as seen above, the rule $x I \mapsto x R$ defines an isomorphism from $\boldsymbol{L}(I)$ onto $\boldsymbol{I}$.

(ii) In the context of (i) above, $\boldsymbol{L}(R) / \boldsymbol{I}=\boldsymbol{L}(R) / \boldsymbol{L}(I) \cong \boldsymbol{L}(R / I)$. The isomorphism $\boldsymbol{L}(R / I) \rightarrow \boldsymbol{L}(R) / \boldsymbol{I}$ is given by $(\lambda+I)(R / I) \mapsto[\lambda R]_{\boldsymbol{I}}$.

(iii) Let $\left\langle L_{i} \mid i \in I\right\rangle$ be a family of coordinatizable lattices and let $\mathcal{F}$ be a filter on $I$. For $i \in I$, let $R_{i}$ be a regular ring such that $\boldsymbol{L}\left(R_{i}\right) \cong L_{i}$. Put $L=\prod_{\mathcal{F}}\left(L_{i} \mid i \in I\right)$ and $R=\prod_{\mathcal{F}}\left(R_{i} \mid i \in I\right)$. It is easy to verify that $\boldsymbol{L}(R)$ is isomorphic to $L$.

The treatment of direct decompositions of a unital ring parallels the theory for bounded lattices. For a unital ring $R$, we denote by cen $R$ the set of all central idempotents of $L$. It is well-known that cen $R$ is a Boolean algebra, with $a \vee b=$ $a+b-a b, a \wedge b=a b$, and $\neg a=1-a$, for all $a, b \in \operatorname{cen} R$. The elements of cen $R$ correspond exactly to the direct decompositions of $R$, in a way that parallels closely Lemma 2.3

There is a natural correspondence between the center of a regular ring $R$ and the center of the lattice $\boldsymbol{L}(R)$, see [20, Satz VI.1.8]. 
Proposition 3.6. Let $R$ be a unital regular ring. The map $e \mapsto e R$ defines an isomorphism from $B=\operatorname{cen} R$ onto $\operatorname{cen} \boldsymbol{L}(R)$. Furthermore,

$$
\operatorname{cen}(e R)=\{x R \mid x \in B\lceil e\} .
$$

\section{Modules}

A right module $E$ over a ring $R$ is semisimple, if the lattice $\operatorname{Sub} E$ of all submodules of $E$ is complemented.

Proposition 4.1. Let $E$ be a semisimple right module over a unital ring $R$. Let $S=$ End $E$ be the endomorphism ring of $E$, and put

$$
\boldsymbol{I}(X)=\{f \in R \mid \operatorname{im} f \subseteq X\} \text {, for all } X \in \operatorname{Sub} E .
$$

Then $S$ is a regular ring and $X \mapsto \boldsymbol{I}(X)$ defines a lattice isomorphism from Sub $E$ onto $\boldsymbol{L}(S)$, with inverse the map $f S \mapsto \operatorname{im} f$.

Proof. Let $f \in S$. Since $E$ is semisimple, there are submodules $X$ and $Y$ of $E$ such that $E=X \oplus \operatorname{ker} f=Y \oplus \operatorname{im} f$. Let $p: E \rightarrow \operatorname{im} f$ be the projection along $Y$. For any $y \in E$, the element $p(y)$ belongs to $\operatorname{im} f$, thus $p(y)=f(x)$ for a unique element $x \in X$, that we denote by $g(y)$. Then $g \in S$ and $f \circ g \circ f=f$, whence $S$ is regular.

Let $X \in \operatorname{Sub} E$. It is clear that $\boldsymbol{I}(X)$ is a right ideal of $S$. Furthermore, since $X$ has a direct summand in $E$, there exists a projection $p$ of $E$ such that $\operatorname{im} p=X$. So, to conclude the proof, it suffices to prove that $\boldsymbol{I}(X)=f S$, for any $f \in S$ with $\operatorname{im} f=X$. It is clear that $f S$ is contained in $\boldsymbol{I}(X)$. Conversely, let $g \in \boldsymbol{I}(X)$. The submodule $\operatorname{ker} f$ of $E$ has a direct summand $Y$. For any $x \in E$, the element $g(x)$ belongs to $X=\operatorname{im} f$, thus there exists a unique $y=h(x)$ in $Y$ such that $g(x)=f(y)$. Hence $h \in S$ and $g=f \circ h$ belongs to $f S$; whence $\boldsymbol{I}(X)=f S$.

In particular, we get the well-known result that for any right vector space $E$ over any division ring, the endomorphism $\operatorname{ring} R=$ End $E$ is regular and $\boldsymbol{L}(R) \cong \operatorname{Sub} E$.

A nontrivial right module $E$ over a ring $R$ is simple, if $\operatorname{Sub} E=\{\{0\}, E\}$. We state the classical Schur's Lemma.

Proposition 4.2. Let $E$ be a simple right module over a ring. Then End $E$ is a division ring.

Let a right module $E$ over a ring $R$ be expressed as a finite direct sum $E=$ $E_{1} \oplus \cdots \oplus E_{n}$. Let $p_{i}$ (resp., $e_{i}$ ) denote the canonical projection on $E_{i}$ (resp., the inclusion map $E_{i} \hookrightarrow E$ ), for all $i \in\{1, \ldots, n\}$. Any endomorphism $f$ of $E$ gives raise to a system of homomorphisms $f_{i, j}: E_{j} \rightarrow E_{i}$, for $i, j \in\{1, \ldots, n\}$, defined as $f_{i, j}=p_{i} \circ f \circ e_{j}$. Then the map

$$
f \mapsto\left(\begin{array}{ccc}
f_{1,1} & \ldots & f_{1, n} \\
\vdots & & \vdots \\
f_{n, 1} & \ldots & f_{n, n}
\end{array}\right)
$$

defines an isomorphism from End $E$ to the ring of all matrices as in the right hand side of (4.1), where $f_{i, j} \in \operatorname{Hom}\left(E_{j}, E_{i}\right)$ for all $i, j \in\{1, \ldots, n\}$, endowed with canonical addition and multiplication. We shall be especially interested in the case where all the $E_{i}$-s are isomorphic submodules. 
Proposition 4.3. In the context above, let $\gamma_{i}: E_{1} \rightarrow E_{i}$ be an isomorphism, for all $i \in\{1, \ldots, n\}$. Then the rule

$$
f \mapsto\left(\begin{array}{ccc}
\gamma_{1}^{-1} f_{1,1} \gamma_{1} & \ldots & \gamma_{1}^{-1} f_{1, n} \gamma_{n} \\
\vdots & & \vdots \\
\gamma_{n}^{-1} f_{n, 1} \gamma_{1} & \ldots & \gamma_{n}^{-1} f_{n, n} \gamma_{n}
\end{array}\right)
$$

defines an isomorphism from End $E$ onto $\mathrm{M}_{n}\left(\right.$ End $\left.E_{1}\right)$.

\section{Coordinatization of Lattices of Length two}

We denote by $M_{X}$ the lattice of length two and distinct atoms $q_{x}$, for $x \in X$, for any nonempty set $X$. The lattices $M_{3}$ and $M_{7}$ are diagrammed on Figure 1, Page 2 Hence the simple lattices of length 2 are exactly the lattices $M_{\kappa}$, where $\kappa \geqslant 3$ is a cardinal number. A bounded lattice $L$ has a spanning $M_{X}$, if there exists a 0,1-lattice homomorphism $f: M_{X} \rightarrow L$ (observe that either $f$ is one-to-one or $L$ is trivial). The following result is folklore.

Proposition 5.1. Let $n \geqslant 3$ be a natural number. Then the following are equivalent:

(i) $n-1$ is a prime power;

(ii) there exists a field $F$ such that $\boldsymbol{L}\left(\mathrm{M}_{2}(F)\right) \cong M_{n}$;

(iii) there exists a regular ring $R$ such that $\boldsymbol{L}(R) \cong M_{n}$;

(iv) there exists a unital ring $R$ such that $\operatorname{Sub}\left(R_{R}\right) \cong M_{n}$;

(v) there are a ring $R$ and a right $R$-module $E$ such that $\operatorname{Sub} E \cong M_{n}$.

Proof. (i) $\Rightarrow$ (ii) Set $q=n-1$. Since the right $\mathbb{F}_{q}$-module $E=\mathbb{F}_{q} \times \mathbb{F}_{q}$ is semisimple, it follows from Proposition 4.1 that $\boldsymbol{L}\left(\mathrm{M}_{2}\left(\mathbb{F}_{q}\right)\right) \cong \operatorname{Sub} E$, whence $\boldsymbol{L}\left(\mathrm{M}_{2}\left(\mathbb{F}_{q}\right)\right)$ is isomorphic to $M_{n}$.

(ii) $\Rightarrow$ (iii) It follows from Proposition 3.1 that $R=\mathrm{M}_{2}(F)$ is a regular ring.

(iii) $\Rightarrow$ (iv) and (iv) $\Rightarrow$ (v) are trivial.

(v) $\Rightarrow$ (i) By assumption, Sub $E=\{\{0\}, E\} \cup\left\{E_{1}, \ldots, E_{n}\right\}$, where $E=E_{i} \oplus E_{j}$ for all distinct $i, j \in\{1, \ldots, n\}$. In particular, $E_{1} \oplus E_{3}=E_{2} \oplus E_{3}=E$, thus $E_{1} \cong$ $E_{2}$. Since Sub $E \cong M_{n}$, the module $E$ is semisimple, whence, by Proposition 4.1 . $S=$ End $E$ is a regular ring and $\operatorname{Sub} E \cong \boldsymbol{L}(S)$. Furthermore, since $E=E_{1} \oplus E_{2}$ and $E_{1} \cong E_{2}$, it follows from Proposition 4.3 that $S \cong \mathrm{M}_{2}(D)$ where we put $D=$ End $E_{1}$. From Sub $E_{1}=\left\{\{0\}, E_{1}\right\}$ and Proposition 4.2 it follows that $D$ is a division ring, and hence, by using again Proposition 4.1]

$$
M_{n} \cong \operatorname{Sub} E \cong \boldsymbol{L}(S) \cong \boldsymbol{L}\left(\mathrm{M}_{2}(D)\right) \cong \operatorname{Sub}(D \times D) .
$$

Therefore, $D$ is a finite division ring, so the order $q$ of $D$ is a prime power, and $n=1+q$.

In particular, the first non-coordinatizable lattice of length two is $M_{7}$, see Figure 1, Page 2

By keeping track of the isomorphisms in the direction $(v) \Rightarrow(\mathrm{i})$ of the proof of Proposition 5.1] we obtain the following additional information.

Proposition 5.2. Let $\kappa$ be a cardinal number greater than or equal to 2. Then the regular rings coordinatizing $M_{1+\kappa}$ are exactly those of the form $\mathrm{M}_{2}(D)$, where $D$ is a division ring with $\kappa$ elements. 
In particular, for any prime power $q$, there exists exactly one regular ring coordinatizing $M_{1+q}$, namely $\mathrm{M}_{2}\left(\mathbb{F}_{q}\right)$.

We denote by $\mathcal{C}$ the class of all coordinatizable lattices and by $\mathcal{N} \mathfrak{C}$ its complement (within, say, the class of all complemented modular lattices). The following consequence of Proposition 5.2 is observed by B. Jónsson in the Introduction of [16].

Corollary 5.3. The class $\mathcal{N} \mathfrak{C}$ is not first-order definable. In particular, $\mathfrak{C}$ is not finitely axiomatizable.

Proof. It follows from Proposition 5.1 that $M_{4 k+7}$ is not coordinatizable, for all $k<\omega$. Let $\mathcal{U}$ be a nonprincipal ultrafilter on $\omega$. The ultraproduct, with respect to $\mathcal{U}$, of the sequence $\left\langle M_{4 k+7} \mid k<\omega\right\rangle$ is isomorphic to $M_{X}$, for some infinite set $X$; thus, by Proposition 5.2 it is coordinatizable. In particular, the class $\mathcal{N} \mathcal{C}$ is not closed under ultraproducts, hence it is not first-order definable.

\section{A First EXAMPLE ABOUT UNIONS OF COORDINATIZABLE LATTICES}

It is well-known that the center $Z(R)$ of a regular ring $R$ is regular (see 8 , Theorem 1.14]). In particular, for each prime $p$, there are $a_{p}, c_{p} \in Z(R)$ with

$$
\begin{aligned}
p^{2} a_{p} & =p \cdot 1_{R}, \\
c_{p} & =1_{R}-p a_{p} .
\end{aligned}
$$

Observe that $c_{p}$ is independent of the element $a_{p}$ satisfying (6.1).

Lemma 6.1. The element $c_{p}$ is a central idempotent of $R$, for each prime $p$. In addition, $c_{p} c_{q}=0$ for all distinct primes $p$ and $q$.

Proof. It is trivial that $c_{p}^{\prime}=p a_{p}$ is idempotent; thus, so is $c_{p}$. As $a_{p}$ is central, so are $c_{p}^{\prime}$ and $c_{p}$.

Now let $p$ and $q$ be distinct primes, and put $e=c_{p} c_{q}$. From $p c_{p}^{\prime} e=p^{2} a_{p} e=p e$, it follows that $p c_{p} e=0$. Since $c_{p} e=e$, we obtain that $p e=0$. Similarly, $q e=0$. Since $p$ and $q$ are coprime, it follows that $e=0$, which establishes our claim.

This makes it possible to solve negatively an open problem raised by B. Jónsson in [17. Section 10].

Proposition 6.2. There exists a countable 2-distributive complemented modular lattice $L$, with a spanning $M_{3}$, which satisfies the two following properties:

(i) $L$ is a directed union of finite coordinatizable lattices;

(ii) $L$ is not coordinatizable.

Consequently, the class $\mathfrak{C}$ of coordinatizable lattices is not closed under countable directed unions.

Proof. Define $L$ as the set of all almost constant sequences $x=\left\langle x_{n} \mid n<\omega\right\rangle$ of elements of $M_{4}$ such that $x(\infty) \in M_{3}$, endowed with componentwise ordering. It is easy to verify that $L$ is a countable 2-distributive complemented modular lattice with a spanning $M_{3}$.

For each $n<\omega$, put $L_{n}=\left(M_{4}\right)^{n} \times M_{3}$, and denote by $f_{n}: L_{n} \rightarrow L$ the map defined by the rule

$$
f_{n}\left(\left\langle x_{0}, \ldots, x_{n-1}, x\right\rangle\right)=\left\langle x_{0}, \ldots, x_{n-1}, x, x, \cdots\right\rangle .
$$


Then $f_{n}$ is a lattice embedding from $L_{n}$ into $L$, and $L$ is the increasing union of all images of the maps $f_{n}$. Observe that each $L_{n}$ (thus each $\left.f_{n}\left[L_{n}\right]\right)$ is coordinatizable, see Proposition 5.1

Now we prove that $L$ is not coordinatizable. Suppose, to the contrary, that there are a regular ring $R$ and an isomorphism $\varepsilon: \boldsymbol{L}(R) \rightarrow L$. For all $n<\omega$, denote by $\pi_{n}: L \rightarrow M_{4}, x \mapsto x(n)$ the $n$-th projection, and put $\pi_{\omega}: L \rightarrow M_{3}, x \mapsto x(\infty)$. Furthermore, put $I_{n}=\pi_{n}^{-1}\{0\}$, for all $n \leqslant \omega$. So $I_{n}$ is a neutral ideal of $L$, and, as $L$ is a complemented modular lattice, $\pi_{n}$ induces an isomorphism from $L / I_{n}$ onto im $\pi_{n}$. The subset $J_{n}=\left\{x \in R \mid \varepsilon(x R) \in I_{n}\right\}$ is a two-sided ideal of $R$, and, by Proposition 3.5 we can define an isomorphism $\varepsilon_{n}: \boldsymbol{L}\left(R / J_{n}\right) \rightarrow L / I_{n}$ by the rule

$$
\varepsilon_{n}\left(\left(\lambda+J_{n}\right)\left(R / J_{n}\right)\right)=[\varepsilon(\lambda R)]_{I_{n}}, \quad \text { for all } \lambda \in R .
$$

In particular, for all $n<\omega, \boldsymbol{L}\left(R / J_{n}\right) \cong M_{4}$, thus, by Proposition 5.2 $R / J_{n} \cong$ $\mathrm{M}_{2}\left(\mathbb{F}_{3}\right)$. Similarly, $R / J_{\omega} \cong \mathrm{M}_{2}\left(\mathbb{F}_{2}\right)$.

Now we consider the central elements $a_{p}, c_{p}$ introduced in (6.1) and 6.2). Projecting the equality $4 a_{2}=2 \cdot 1_{R}$ on $R / J_{n}$, for $n \leqslant \omega$, yields

$$
\begin{aligned}
& c_{2} \in J_{n}, \text { for all } n<\omega, \\
& c_{2} \in 1+J_{\omega} .
\end{aligned}
$$

From $\bigcap_{n<\omega} I_{n}=\{0\}$ it follows easily that $\bigcap_{n<\omega} J_{n}=\{0\}$, so (6.3) yields that $c_{2}=0$, which contradicts (6.4).

\section{Determining Sequences and Atomic Boolean Algebras}

For models $A$ and $B$ of a first-order language $\mathcal{L}$, let $A \equiv B$ denote elementary equivalence of $A$ and $B$. A Boolean algebra $B$ is atomic, if the unit element of $B$ is the join of the set At $B$ of all atoms of $B$. The following lemma is an immediate application of A. Tarski's classification of the complete extensions of the theory of Boolean algebras (see C. C. Chang and H. J. Keisler [5, Section 5.5]).

Lemma 7.1. Let $A$ and $B$ be atomic Boolean algebras. Then $A \equiv B$ iff $\min \left\{|\operatorname{At} A|, \aleph_{0}\right\}=\min \left\{\mid\right.$ At $\left.B \mid, \aleph_{0}\right\}$.

Now we recall a few notions about Boolean products. Let $\mathcal{L}$ be a first-order language, let $X$ be a Boolean space, and let $A$ be a subdirect product of a family $\left\langle A_{\mathfrak{p}} \mid \mathfrak{p} \in X\right\rangle$ of models of $\mathcal{L}$. For a first-order formula $\varphi\left(\mathrm{x}_{0}, \ldots, \mathrm{x}_{n-1}\right)$ of $\mathcal{L}$ and elements $a_{0}, \ldots, a_{n-1} \in A$, we put

$$
\left\|\varphi\left(a_{0}, \ldots, a_{n-1}\right)\right\|=\left\{\mathfrak{p} \in X \mid A_{\mathfrak{p}} \models \varphi\left(a_{0}(\mathfrak{p}), \ldots, a_{n-1}(\mathfrak{p})\right)\right\} .
$$

We say that the subdirect product

$$
A \hookrightarrow \prod_{\mathfrak{p} \in X} A_{\mathfrak{p}}
$$

is a Boolean product (see [4. Section IV.8]), if the following conditions hold:

(i) $\|\varphi\|$ belongs to Clop $X$, for every atomic sentence $\varphi$ with parameters from $A$;

(ii) for any elements $a, b \in A$ and any clopen subset $Y$ of $X$, the element $a \uparrow_{Y} \cup b \uparrow_{X \backslash Y}$ belongs to $A$. 
If, in addition, the Boolean value $\|\varphi\|$ belongs to Clop $X$, for every $\mathcal{L}$-sentence $\varphi$ with parameters from $A$, we say that (7.1) is a strong Boolean product. It is observed in M. Weese [25, Section 8] that the statement that (7.1) is a strong Boolean product follows from the so-called maximality property, that is, for every $\mathcal{L}$-formula $\varphi\left(\mathrm{x}, \mathrm{y}_{0}, \ldots, \mathrm{y}_{n-1}\right)$ and all $b_{0}, \ldots, b_{n-1} \in A$, there exists $a \in A$ such that

$$
\left\|\varphi\left(a, b_{0}, \ldots, b_{n-1}\right)\right\|=\left\|\exists \times \varphi\left(x, b_{0}, \ldots, b_{n-1}\right)\right\| .
$$

As the following easy lemma shows, the two notions are, in fact, equivalent.

Lemma 7.2. Any strong Boolean product has the maximality property.

Proof. Suppose that (7.1) is a strong Boolean product, let $\varphi\left(\mathrm{x}, \mathrm{y}_{0}, \ldots, \mathrm{y}_{n-1}\right)$ be a $\mathcal{L}$-formula, and let $b_{0}, \ldots, b_{n-1} \in A$. It follows from the assumption that $U=$ $\left\|\exists \mathrm{x} \varphi\left(\mathrm{x}, b_{0}, \ldots, b_{n-1}\right)\right\|$ is a clopen subset of $X$. By definition of the $\left\|_{-}\right\|$symbol, the equality

$$
U=\bigcup\left(\left\|\varphi\left(x, b_{0}, \ldots, b_{n-1}\right)\right\| \mid x \in A\right)
$$

holds, thus, since $U$ is compact, there are $k<\omega$ and elements $a_{0}, \ldots, a_{k-1} \in A$ such that

$$
U=\bigcup\left(\left\|\varphi\left(a_{j}, b_{0}, \ldots, b_{n-1}\right)\right\| \mid j<k\right) .
$$

There are pairwise disjoint clopen subsets $U_{j} \subseteq\left\|\varphi\left(a_{j}, b_{0}, \ldots, b_{n-1}\right)\right\|$, for $j<k$, such that $U=\bigcup\left(U_{j} \mid j<k\right)$. Since (7.1) is a Boolean product, there exists $a \in A$ such that $\left.a\right|_{U_{j}}=a_{j} \uparrow_{U_{j}}$, for all $j<k$. Therefore,

$$
U=\left\|\varphi\left(a, b_{0}, \ldots, b_{n-1}\right)\right\| .
$$

The following definition is the natural extension of S. Feferman and R. L. Vaught's determining sequences (see [5, Section 6.3]) to strong Boolean products.

Definition 7.3. For a formula $\varphi$ of a first-order language $\mathcal{L}$, a pair $\left\langle\Phi,\left\langle\varphi_{i} \mid i \in I\right\rangle\right\rangle$ is a determining sequence of $\varphi$, if the following conditions hold:

(i) the set $I$ is finite, $\Phi$ is a first-order formula of the language $\langle\vee, \wedge\rangle$ with set of free variables indexed by $I$, and all $\varphi_{i}$-s are $\mathcal{L}$-formulas with the same free variables as $\varphi$;

(ii) $\Phi$ is isotone, that is, the theory of Boolean algebras infers the following statement:

$$
\left[\Phi\left(\mathrm{x}_{i} \mid i \in I\right) \text { and } \bigwedge_{i \in I}\left(\mathrm{x}_{i} \leq \mathrm{y}_{i}\right)\right] \Longrightarrow \Phi\left(\mathrm{y}_{i} \mid i \in I\right) .
$$

(iii) for every strong Boolean product as in (7.1) and for every $\mathcal{L}$-formula $\varphi(\vec{a})$ with parameters from $A$, the following equivalence holds:

$$
\left.A \models \varphi(\vec{a}) \Longleftrightarrow \operatorname{Clop} X \models \Phi\left(\left\|\varphi_{i}(\vec{a})\right\| \mid i \in I\right)\right) .
$$

An immediate consequence of Lemma 7.2 and [25] Theorem 8.1] is the following.

Lemma 7.4. For every first-order language $\mathcal{L}$, every formula of $\mathcal{L}$ has a determining sequence.

We shall use later the following application to Boolean algebras.

Lemma 7.5. Let $A$ be a subalgebra of a Boolean algebra $B$. We suppose that both $A$ and $B$ are atomic, with $\operatorname{At} A=\operatorname{At} B$. Then $A$ is an elementary submodel of $B$. 
Proof. Let $\varphi\left(\mathrm{x}_{0}, \ldots, \mathrm{x}_{n-1}\right)$ be a formula of the language $\langle\vee, \wedge\rangle$ and let $a_{0}, \ldots$, $a_{n-1} \in A$ such that $A \models \varphi(\vec{a})$ (where $\vec{a}=\left\langle a_{0}, \ldots, a_{n-1}\right\rangle$ ); we shall prove that $B \models \varphi(\vec{a})$. Denote by $U$ the (finite) set of atoms of the Boolean subalgebra of $A$ generated by $\left\{a_{i} \mid i<n\right\}$. We use the canonical isomorphisms

$$
A \cong \prod(A|u| u \in U), \quad B \cong \prod(B \nmid u \mid u \in U) .
$$

Let $\mathcal{L}$ denote the first-order language obtained by enriching the language of Boolean algebras by $n$ additional constants $\underline{a}_{0}, \ldots, \underline{a}_{n-1}$. Let $\bar{\varphi}$ denote the sentence $\varphi\left(\underline{a}_{0}, \ldots, \underline{a}_{n-1}\right)$ of $\mathcal{L}$. The assumption that $A \models \varphi(\vec{a})$ can be rewritten as

$$
\prod(\langle A\lceil u, \vec{a} \wedge u\rangle| u \in U) \models \bar{\varphi} .
$$

Let $u \in U$. Since $a_{i} \wedge u \in\{0, u\}$ for all $i<n$, it follows from Lemma 7.1 that $\langle A\lceil u, \vec{a} \wedge u\rangle \equiv\langle B\lceil u, \vec{a} \wedge u\rangle$. Therefore, since elementary equivalence is preserved under direct products (see [5] Theorem 6.3.4]), it follows from (7.2) that

$$
\prod(\langle B \mid u, \vec{a} \wedge u\rangle \mid u \in U) \models \bar{\varphi},
$$

that is, $B \models \varphi(\vec{a})$.

\section{CoORdinatizaBILITY IS NOT FIRST-ORDER}

We put $P_{p}=\left\{p^{k !} \mid k \in \mathbb{N}\right\}$ (where $k !=k(k-1) \cdots 2 \cdot 1$ ), for any prime $p$, and we put $P=P_{2} \cup P_{3}$. We shall construct a pair of lattices $K$ and $L$. The construction can also be performed in a similar fashion for any pair of distinct primes, we just pick 2 and 3 for simplicity. Our lattices are the following:

$$
\begin{aligned}
K & =\left\{x \in \prod\left(M_{1+k} \mid k \in P\right) \mid x \text { is almost constant }\right\} ; \\
L & =\left\{x \in \prod\left(M_{1+k} \mid k \in P\right) \mid \text { both } x \uparrow_{P_{2}} \text { and } x \uparrow_{P_{3}} \text { are almost constant }\right\} .
\end{aligned}
$$

Of course, both $K$ and $L$ are 2-distributive complemented modular lattices with spanning $M_{3}$, and $K$ is a 0,1 -sublattice of $L$. Furthermore, cen $K=K \cap \mathbf{2}^{P}$ and cen $L=L \cap \mathbf{2}^{P}$, where $\mathbf{2}=\{0,1\}$. Let $\infty, \infty_{2}$, and $\infty_{3}$ denote distinct objects not in $P$. We put

$$
U=P \cup\{\infty\}, \quad V=P \cup\left\{\infty_{2}, \infty_{3}\right\} .
$$

Endow $U$ with the least topology making every singleton in $P$ clopen, and $V$ with the least topology making every singleton of $P$ clopen as well as $P_{2} \cup\left\{\infty_{2}\right\}$ (and thus also $P_{3} \cup\left\{\infty_{3}\right\}$ ). Observe that $U$ is isomorphic to $\omega+1$ endowed with its interval topology, while $V$ is isomorphic to the disjoint union of two copies of $U$. In particular, both $U$ and $V$ are Boolean spaces. The canonical map from $V$ onto $U$ is the map $e: V \rightarrow U$, whose restriction to $P$ is the identity, and that sends both $\infty_{2}$ and $\infty_{3}$ to $\infty$. The inverse map $\varepsilon$ : Clop $U \hookrightarrow \operatorname{Clop} V, X \mapsto e^{-1}[X]$ is the canonical embedding from Clop $U$ into Clop $V$. As an immediate application of Lemma 7.5. we observe the following.

Lemma 8.1. The map $\varepsilon$ is an elementary embedding from Clop $U$ into Clop $V$.

Now we shall represent both $K$ and $L$ as Boolean products. We put

$$
\begin{aligned}
K^{\prime} & =\left\{x \in \mathbf{C}\left(U, M_{\omega}\right) \mid(\forall k \in P) x(k) \in M_{1+k}\right\} ; \\
L^{\prime} & =\left\{x \in \mathbf{C}\left(V, M_{\omega}\right) \mid(\forall k \in P) x(k) \in M_{1+k}\right\} .
\end{aligned}
$$

The verification of the following lemma is trivial. 
Lemma 8.2. Both maps from $K^{\prime}$ to $K$ and from $L^{\prime}$ to $L$ defined by restriction to $P$ are lattice isomorphisms.

We set $M_{1+k}=M_{\omega}$ for $k \in\left\{\infty, \infty_{2}, \infty_{3}\right\}$. With each of the lattices $K^{\prime}$ and $L^{\prime}$ is associated a subdirect product, namely,

$$
\begin{aligned}
K^{\prime} \hookrightarrow \prod\left(M_{1+k} \mid k \in U\right), & x \mapsto\langle x(k) \mid k \in U\rangle \\
L^{\prime} \hookrightarrow \prod\left(M_{1+k} \mid k \in V\right), & x \mapsto\langle x(k) \mid k \in V\rangle .
\end{aligned}
$$

We denote by $\left\|_{-}\right\|^{K}$ (resp., $\left\|_{-}\right\|^{L}$ ) the Boolean value function defined by the subdirect decomposition (8.1) (resp., (8.2)). We denote by $a^{\prime}$ (resp., $a^{\prime \prime}$ ) the image of $a$ under the canonical isomorphism from $K$ onto $K^{\prime}$ (resp., from $L$ onto $L^{\prime}$ ), for any $a \in K$ (resp., $a \in L$ ).

Lemma 8.3. Both subdirect products (8.1) and (8.2) are strong Boolean products. Furthermore, $\left\|\varphi\left(\vec{a}^{\prime \prime}\right)\right\|^{L}=\varepsilon\left(\left\|\varphi\left(\vec{a}^{\prime}\right)\right\|^{K}\right)$, for every formula $\varphi\left(\mathrm{x}_{0}, \ldots, \mathrm{x}_{n-1}\right)$ of $\langle\vee, \wedge\rangle$ and all $a_{0}, \ldots, a_{n-1} \in K$.

Proof. Let $\varphi\left(\mathrm{x}_{0}, \ldots, \mathrm{x}_{n-1}\right)$ be a formula of the language $\langle\vee, \wedge\rangle$. An easy application of the Compactness Theorem of first-order predicate logic gives that for any $a_{0}, \ldots$, $a_{n-1} \in M_{\omega}$, the following statements are equivalent:

- $M_{\omega} \models \varphi\left(a_{0}, \ldots, a_{n-1}\right)$;

- $M_{1+k}=\varphi\left(a_{0}, \ldots, a_{n-1}\right)$ for all but finitely many $k \in P$;

- $M_{1+k} \models \varphi\left(a_{0}, \ldots, a_{n-1}\right)$ for infinitely many $k \in P$.

Hence, for any finite sequence $\vec{a}=\left\langle a_{i} \mid i<n\right\rangle$ in $K^{n}$, both Boolean values $\left\|\varphi\left(\vec{a}^{\prime}\right)\right\|^{K}$ and $\left\|\varphi\left(\vec{a}^{\prime \prime}\right)\right\|^{L}$ are clopen, respectively in $U$ and in $V$, and they are determined by their restrictions to $P$. Furthermore, $\left\|\varphi\left(\vec{a}^{\prime \prime}\right)\right\|^{L}=\varepsilon\left(\left\|\varphi\left(\vec{a}^{\prime}\right)\right\|^{K}\right)$.

Proposition 8.4. The lattice $K$ is an elementary submodel of $L$.

Proof. Let $\varphi\left(\mathrm{x}_{0}, \ldots, \mathrm{x}_{m-1}\right)$ be a formula of $\langle\vee, \wedge\rangle$ and let $\vec{a}=\left\langle a_{i} \mid i<m\right\rangle \in$ $K^{m}$ such that $K \models \varphi(\vec{a})$. By Lemma $7.4 \varphi$ has a determining sequence, say, $\left\langle\Phi,\left\langle\varphi_{j} \mid j\langle n\rangle\right\rangle\right.$. Since $K^{\prime} \models \varphi\left(\vec{a}^{\prime}\right)$ and by Lemma 8.3 the following relation holds:

$$
\text { Clop } U \models \Phi\left(\left\|\varphi_{0}\left(\vec{a}^{\prime}\right)\right\|^{K}, \ldots,\left\|\varphi_{n-1}\left(\vec{a}^{\prime}\right)\right\|^{K}\right) .
$$

Hence, by Lemma 8.1

$$
\text { Clop } V \models \Phi\left(\varepsilon\left(\left\|\varphi_{0}\left(\vec{a}^{\prime}\right)\right\|^{K}\right), \ldots, \varepsilon\left(\left\|\varphi_{n-1}\left(\vec{a}^{\prime}\right)\right\|^{K}\right)\right) \text {. }
$$

By Lemma $8.3 \varepsilon\left(\left\|\varphi_{j}\left(\vec{a}^{\prime}\right)\right\|^{K}\right)=\left\|\varphi_{j}\left(\vec{a}^{\prime \prime}\right)\right\|^{L}$, for all $j<n$, and hence, again by Lemma 8.3, $L^{\prime} \models \varphi\left(\vec{a}^{\prime \prime}\right)$, and therefore $L \models \varphi(\vec{a})$.

Proposition 8.5. The lattice $K$ is not coordinatizable.

Proof. Suppose otherwise, and let $\varepsilon: \boldsymbol{L}(R) \rightarrow K$ be an isomorphism, where $R$ is a regular ring. We denote by $\pi_{q}: K \rightarrow M_{1+q}$ the canonical projection, for all $q \in P$. The subset $I_{q}=\pi_{q}^{-1}\{0\}$ is a neutral ideal of $K$, and, as $K$ is a complemented modular lattice, $\pi_{q}$ induces an isomorphism from $K / I_{q}$ onto $M_{1+q}$. The subset $J_{q}=\left\{x \in R \mid \varepsilon(x R) \in I_{q}\right\}$ is a two-sided ideal of $R$, and, by Proposition 3.5 we can define an isomorphism $\varepsilon_{q}: \boldsymbol{L}\left(R / J_{q}\right) \rightarrow K / I_{q}$ by the rule

$$
\varepsilon_{q}\left(\left(\lambda+J_{q}\right)\left(R / J_{q}\right)\right)=[\varepsilon(\lambda R)]_{I_{q}}, \quad \text { for all } \lambda \in R .
$$


In particular, for all $q \in P, \boldsymbol{L}\left(R / J_{q}\right) \cong M_{1+q}$, thus, by Proposition 5.2

$$
R / J_{q} \cong \mathrm{M}_{2}\left(\mathbb{F}_{q}\right) \text {. }
$$

Now we consider again the elements $a_{p}$ and $c_{p}$ introduced in (6.1) and (6.2). It follows from Proposition [3.6 that $u_{p}=\varepsilon\left(c_{p} R\right)$ belongs to the center of $K$. From $c_{2} c_{3}=0$ (see Lemma 6.1) it follows that $u_{2} \wedge u_{3}=0$. As cen $K$ consists of all almost constant elements of $\{0,1\}^{P}$, it follows that either $u_{2}(\infty)=0$ or $u_{3}(\infty)=0$. Suppose, for example, that $u_{2}(\infty)=0$. In particular, there exists $q \in P_{2}$ such that $\pi_{q}\left(u_{2}\right)=0$. As $q$ is a power of 2 and by $(8.3)$, we get $2 a_{2} \in J_{q}$, so

$$
c_{2} \in 1+J_{q} \text {. }
$$

On the other hand,

$$
\begin{aligned}
0 & =\left[u_{2}\right]_{I_{q}} & & \text { (because } \left.\pi_{q}\left(u_{2}\right)=0\right) \\
& =\left[\varepsilon\left(c_{2} R\right)\right]_{I_{q}} & & \text { (by the definition of } \left.u_{2}\right) \\
& =\varepsilon_{q}\left(\left(c_{2}+J_{q}\right)\left(R / J_{q}\right)\right) & & \text { (by the definition of } \left.\varepsilon_{q}\right),
\end{aligned}
$$

thus, as $\varepsilon_{q}$ is an isomorphism, $c_{2} \in J_{q}$, which contradicts (8.4).

Proposition 8.6. The lattice $L$ is coordinatizable.

Proof. It is obvious that $L \cong L_{2} \times L_{3}$, where we put

$$
L_{p}=\left\{x \in \prod\left(M_{1+q} \mid q \in P_{p}\right) \mid x \text { is almost constant }\right\}, \quad \text { for each prime } p .
$$

Hence it suffices to prove that $L_{p}$ is coordinatizable, for each prime $p$.

Put $S_{q}=\mathrm{M}_{2}\left(\mathbb{F}_{q}\right)$, for each prime power $q$. As $\mathbb{F}_{p^{k}}$ ! is a subfield of $\mathbb{F}_{p^{(k+1)}}$ ! for each $k<\omega$, we can define a unital ring $R_{p}$ by

$$
R_{p}=\left\{\lambda \in \prod\left(S_{q} \mid q \in P_{p}\right) \mid \lambda \text { is almost constant }\right\} .
$$

It is easy to verify that $R_{p}$ is a regular ring. We shall prove that $L_{p} \cong \boldsymbol{L}\left(R_{p}\right)$. Fix a one-to-one enumeration $\left\langle\xi_{k} \mid k \in \mathbb{N}\right\rangle$ of $\mathbb{F}_{p^{\infty}}$ such that $\mathbb{F}_{p^{n !}}=\left\{\xi_{k} \mid 1 \leqslant k \leqslant p^{n !}\right\}$ for all $n<\omega$. We put $\alpha_{0}=\left(\begin{array}{ll}0 & 0 \\ 0 & 1\end{array}\right)$ and $\alpha_{k}=\left(\begin{array}{cc}1 & 0 \\ \xi_{k} & 0\end{array}\right)$, for each $k \in \mathbb{N}$. For all $q \in P_{p}$, there exists a unique isomorphism $\eta_{q}: \boldsymbol{L}\left(S_{q}\right) \rightarrow M_{1+q}$ such that

$$
\eta_{q}\left(\alpha_{k} S_{q}\right)=q_{k} \quad\left(\text { the } k \text {-th atom of } M_{1+q}\right), \quad \text { for all } k \in\{0,1, \ldots, q\} .
$$

We can define a map $\varepsilon_{p}: \boldsymbol{L}\left(R_{p}\right) \rightarrow \prod\left(M_{1+q} \mid q \in P_{p}\right)$ by the rule

$$
\varepsilon_{p}\left(\lambda R_{p}\right)=\left\langle\eta_{q}\left(\lambda_{q} S_{q}\right) \mid q \in P_{p}\right\rangle, \quad \text { for all } \lambda \in R_{p} .
$$

For any $\lambda \in R_{p}$, there exists $m \in P_{p}$ such that $\lambda_{q}=\lambda_{m}$ for all $q \geqslant m$ in $P_{p}$. If $\lambda_{m}$ is neither zero nor invertible in $S_{m}$, then there exists a unique $k \in\{0,1, \ldots, m\}$ such that $\lambda_{m} S_{m}=\alpha_{k} S_{m}$, thus $\lambda_{q} S_{q}=\alpha_{k} S_{q}$ for all $q \geqslant m$ in $P_{p}$, and thus $\varepsilon_{p}\left(\lambda R_{p}\right)$ is almost constant (with limit $q_{k}$ ). This holds trivially in case $\lambda_{m}$ is either zero or invertible, therefore the range of $\varepsilon_{p}$ is contained in $L_{p}$. Now it follows from Corollary 3.4(ii) that $\varepsilon_{p}$ is a lattice homomorphism from $\boldsymbol{L}\left(R_{p}\right)$ onto $L_{p}$.

For idempotent $\alpha, \beta \in R_{p}$, if $\varepsilon_{p}\left(\alpha R_{p}\right)=\varepsilon_{p}\left(\beta R_{p}\right)$, then $\eta_{q}\left(\alpha_{q} S_{q}\right)=\eta_{q}\left(\beta_{q} S_{q}\right)$ for all $q \in P_{p}$, thus (as the $\eta_{q}$ s are isomorphisms) $\alpha_{q}=\beta_{q} \alpha_{q}$ and $\beta_{q}=\alpha_{q} \beta_{q}$ for all $q \in P_{p}$, so $\alpha=\beta \alpha$ and $\beta=\alpha \beta$, and so $\alpha R_{p}=\beta R_{p}$. Therefore, $\varepsilon_{p}$ is one-to-one.

Let $x \in L_{p}$. If $x(\infty) \in\{0,1\}$, then, as each $\eta_{q}$ is an isomorphism, there exists $\lambda \in R_{p}$, with limit either 0 or 1 , such that $\varepsilon_{p}\left(\lambda R_{p}\right)=x$. Now suppose that $x(\infty)=q_{k}$, with $k<\omega$. There exists $m \geqslant k$ in $P_{p}$ such that $x_{q}=q_{k}$ holds for all 
$q \geqslant m$ in $P_{p}$. For each $q<m$ in $P_{p}$, there exists $\lambda_{q} \in S_{q}$ such that $\eta_{q}\left(\lambda_{q} S_{q}\right)=x_{q}$. Put $\lambda_{q}=\alpha_{k}$, for all $q \geqslant m$ in $P_{p}$. Then $\lambda \in R_{p}$ and $\varepsilon_{p}\left(\lambda R_{p}\right)=x$. Therefore, the map $\varepsilon_{p}$ is surjective, and so it is an isomorphism.

By combining Propositions 8.4 8.5 and 8.6 we obtain a negative solution to Jónsson's Problem.

Theorem 8.7. Neither the class $\mathfrak{C}$ of coordinatizable lattices nor its complement $\mathfrak{N} \mathfrak{C}$ are first-order classes. In fact, there are countable, 2-distributive lattices $K$ and $L$ with spanning $M_{3}$ such that $K$ is an elementary sublattice of $L$, the lattice $K$ belongs to $\mathcal{N} \mathcal{C}$, and the lattice $L$ belongs to $\mathcal{C}$.

By using sheaf-theoretical methods, we could prove that every countable 2distributive complemented modular lattice with a spanning $M_{\omega}$ is coordinatizable. Hence the use of prime numbers in Theorem 8.7 is somehow unavoidable. As we shall see in the next section, this result does not extend to the uncountable case.

\section{An uncountable NON-COORDinAtizable lattice With A SPANning $M_{\omega}$}

We start with an elementary lemma of linear algebra.

Lemma 9.1. Let $E$ be a unital ring, let $F$ be a division ring, let $n \in \mathbb{N}$, and let $\varphi: \mathrm{M}_{n}(E) \rightarrow \mathrm{M}_{n}(F)$ be a unital ring homomorphism. There are a unital ring homomorphism $\sigma: E \rightarrow F$ and a matrix $a \in \mathrm{GL}_{n}(F)$ such that

$$
\varphi(x)=a(\sigma x) a^{-1}, \text { for all } x \in \mathrm{M}_{n}(E),
$$

where $\sigma x$ denotes the matrix obtained by applying $\sigma$ to all the entries of $x$.

Proof. Put $R=\mathrm{M}_{n}(E)$ and $S=\mathrm{M}_{n}(F)$. Let $\left\langle e_{i, j}^{E} \mid 1 \leqslant i, j \leqslant n\right\rangle$ denote the canonical system of matrix units of $R$, and similarly for $S$. Then $\left\langle\varphi\left(e_{i, j}^{E}\right) \mid 1 \leqslant i, j \leqslant n\right\rangle$ is a system of matrix units of $S$, thus, since $F$ is a division ring, there exists $a \in \mathrm{GL}_{n}(F)$ such that $\varphi\left(e_{i, j}^{E}\right)=a e_{i, j}^{F} a^{-1}$ for all $i, j \in\{1, \ldots, n\}$. Hence, conjugating $\varphi$ by $a^{-1}$, we reduce the problem to the case where $\varphi\left(e_{i, j}^{E}\right)=e_{i, j}^{F}$, for all $i, j \in\{1, \ldots, n\}$. Fix $i, j \in\{1, \ldots, n\}$ and $x \in E$. As $e_{i, j}^{E} x=e_{i, i}^{E} x e_{j, j}^{E}$, the value $\varphi\left(e_{i, j}^{E} x\right)$ belongs to $e_{i, i}^{F} S e_{j, j}^{F}$, thus it has the form $e_{i, j}^{F} \sigma_{i, j}(x)$, for a unique $\sigma_{i, j}(x) \in F$.

It is obvious that all $\sigma_{i, j}$-s are unit-preserving additive homomorphisms from $E$ to $F$. Applying the ring homomorphism $\varphi$ to the equalities

$$
e_{i, i}^{E} x=e_{i, j}^{E}\left(e_{j, i}^{E} x\right)=\left(e_{i, j}^{E} x\right) e_{j, i}^{E} \text {, for all } x \in E,
$$

we obtain that $\sigma_{i, i}=\sigma_{i, j}=\sigma_{j, i}$. Thus $\sigma_{i, j}=\sigma_{1,1}$, for all $i, j \in\{1, \ldots, n\}$. Denote this map by $\sigma$. So $\varphi(x)=\sigma x$, for all $x \in R$, and $\sigma$ is a ring homomorphism.

Corollary 9.2. Let $E$ and $F$ be division rings and let $\varphi: \mathrm{M}_{2}(E) \hookrightarrow \mathrm{M}_{2}(F)$ be a unital ring embedding. If $\varphi$ is not an isomorphism, then the complement of the image of $\boldsymbol{L}(\varphi)$ in $\boldsymbol{L}\left(\mathrm{M}_{2}(F)\right)$ has cardinality at least $|E|$.

Proof. By using Lemma 9.1 we may conjugate $\varphi$ by a suitable element of $\mathrm{M}_{2}(F)$ to reduce the problem to the case where $\varphi(x)=\sigma x$ identically on $\mathrm{M}_{2}(E)$, for a suitable unital ring embedding $\sigma: E \hookrightarrow F$. Since $\varphi$ is not an isomorphism, $\sigma$ is not surjective. In particular, $|F \backslash \sigma E| \geqslant|E|$. Now observe that the matrices of the form $e_{\lambda}=\left(\begin{array}{ll}1 & 0 \\ \lambda & 0\end{array}\right)$, with $\lambda \in F$, are idempotent matrices of $\mathrm{M}_{2}(F)$ with pairwise distinct image spaces. Furthermore, $e_{\lambda} \mathrm{M}_{2}(F)$ does not belong to the image of $\boldsymbol{L}(\varphi)$, for all $\lambda \in F \backslash \sigma E$. 
Now our counterexample. For an infinite cardinal number $\kappa$, whose successor cardinal we denote by $\kappa^{+}$, we put

$$
\begin{aligned}
& \bar{L}_{\kappa}=\left\{x \in\left(M_{\kappa+1}\right)^{\kappa^{+}} \mid x \text { is almost constant }\right\}, \\
& L_{\kappa}=\left\{x \in \bar{L}_{\kappa} \mid x(\infty) \in M_{\kappa}\right\},
\end{aligned}
$$

both ordered componentwise. It is obvious that $L_{\kappa}$ is a 2-distributive complemented modular lattice with a spanning $M_{\kappa}$.

Theorem 9.3. The lattice $L_{\kappa}$ is not coordinatizable, for any infinite cardinal number $\kappa$.

Proof. Otherwise let $R$ be a unital regular ring and let $\varepsilon: \boldsymbol{L}(R) \rightarrow L_{\kappa}$ be an isomorphism. For $x \in M_{\kappa+1}$ and $i<\kappa^{+}$, let $x \cdot u_{i}$ denote the element of $L_{\kappa}$ with $i$-th component $x$ and all other components zero; furthermore, put $u_{i}=1 \cdot u_{i}$. There are central idempotents $a_{i} \in \operatorname{cen} R$ such that $\varepsilon\left(a_{i} R\right)=u_{i}$, for all $i<\kappa^{+}$. Since $\boldsymbol{L}\left(a_{i} R\right) \cong L_{\kappa}\left\lceil u_{i} \cong M_{\kappa}\right.$ (see Proposition 3.5 (i)), there exists, by Proposition [5.2 a division ring $E_{i}$ of cardinality $\kappa$ such that $a_{i} R \cong \mathrm{M}_{2}\left(E_{i}\right)$ (as rings). Put

$$
J_{X}=\bigoplus\left(a_{i} R \mid i \in X\right), \text { for all } X \subseteq \kappa^{+},
$$

and put $J=J_{\kappa^{+}}$. Observe that $I=\varepsilon \boldsymbol{L}(J)$ is the ideal of all almost null elements of $L_{\kappa}$. It follows that

$$
\boldsymbol{L}(R / J) \cong \boldsymbol{L}(R) / \boldsymbol{L}(J) \cong L_{\kappa} / I \cong M_{\kappa},
$$

with the canonical isomorphism $\zeta: \boldsymbol{L}(R / J) \rightarrow L_{\kappa} / I$ of Proposition 3.5(ii) given by

$$
\zeta((x+J) R / J)=[\varepsilon(x R)]_{I}, \text { for all } x \in R .
$$

Furthermore, it follows from (9.3) and Proposition 5.2 that $R / J \cong \mathrm{M}_{2}(E)$, for some division ring $E$ with $\kappa$ elements. In particular, $R / J$ has $\kappa$ elements. For any $\lambda \in R / J$, pick $\dot{\lambda} \in R$ such that $\lambda=\dot{\lambda}+J$. Of course, we may take $\dot{0}_{R / J}=0_{R}$ and $\dot{1}_{R / J}=1_{R}$. For $\alpha, \beta, \gamma \in R / J$ such that $\gamma=\alpha-\beta$, there exists a finite subset $X$ of $\kappa^{+}$such that $\dot{\gamma} \equiv \dot{\alpha}-\dot{\beta}\left(\bmod J_{X}\right)$. By doing the same for the product map $\langle\alpha, \beta\rangle \mapsto \alpha \beta$, the zero, and the unit of $R / J$ and forming the union of all corresponding $X$-s, we obtain a subset $X$ of $\kappa^{+}$of cardinality at most $\kappa$ such that $p_{i}: \lambda \mapsto a_{i} \dot{\lambda}$ defines a unital ring homomorphism from $R / J$ to $a_{i} R$, for all $i \in \kappa^{+} \backslash X$. Since $R / J$ is simple, $p_{i}$ is an embedding.

Put $\dot{x}_{\lambda}=\varepsilon(\dot{\lambda} R)\left(\right.$ an element of $\left.L_{\kappa}\right)$, for all $\lambda \in R / J$. Observe that $\zeta(\lambda(R / J))=$ $\left[\dot{x}_{\lambda}\right]_{I}$, for all $\lambda \in R / J$; in particular

$$
L_{\kappa} / I=\left\{\left[\dot{x}_{\lambda}\right]_{I} \mid \lambda \in R / J\right\} .
$$

For $\alpha, \beta, \gamma \in R / J$ such that $\left[\dot{x}_{\gamma}\right]_{I}=\left[\dot{x}_{\alpha}\right]_{I} \vee\left[\dot{x}_{\beta}\right]_{I}$, there exists a finite subset $Y$ of $\kappa^{+}$such that the equality $\dot{x}_{\gamma}(i)=\dot{x}_{\alpha}(i) \vee \dot{x}_{\beta}(i)$ holds for all $i \in \kappa^{+} \backslash Y$. By doing the same for the meet and the constants 0 and 1, and then taking the union of $X$ and all corresponding $Y$-s, we obtain a subset $Y$ of $\kappa^{+}$containing $X$, with at most $\kappa$ elements, such that $g_{i}:\left[\dot{x}_{\lambda}\right]_{I} \mapsto \dot{x}_{\lambda} \wedge u_{i}$ defines a $\langle\vee, \wedge, 0,1\rangle$-homomorphism from $L_{\kappa} / I$ into $L_{\kappa} \backslash u_{i}$, for all $i \in \kappa^{+} \backslash Y$; since $L_{\kappa} / I$ is simple, $g_{i}$ is, actually, an embedding. 
Let $\zeta_{i}: \boldsymbol{L}\left(a_{i} R\right) \rightarrow L_{\kappa}\left\lceil u_{i}, x R \mapsto \varepsilon(x R)\right.$ denote the canonical isomorphism, for all $i<\kappa^{+}$. We shall verify that the following diagram is commutative, for $i \in \kappa^{+} \backslash Y$.

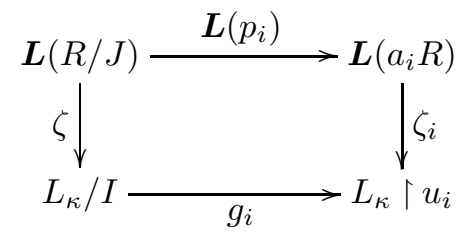

Let $\lambda \in R / J$. It is immediate that $\zeta_{i} \circ \boldsymbol{L}\left(p_{i}\right)(\lambda(R / J))=\varepsilon\left(a_{i} \dot{\lambda} R\right)$. On the other hand, we compute

$$
\begin{aligned}
g_{i} \circ \zeta(\lambda(R / J)) & =g_{i}\left(\left[\dot{x}_{\lambda}\right]_{I}\right) \\
& =\dot{x}_{\lambda} \wedge u_{i} \\
& =\varepsilon(\dot{\lambda} R) \wedge \varepsilon\left(a_{i} R\right) \\
& =\varepsilon\left(\dot{\lambda} R \wedge a_{i} R\right) \\
& \left.=\varepsilon\left(a_{i} \dot{\lambda} R\right) \quad \text { (because } a_{i} \in \operatorname{cen} R\right),
\end{aligned}
$$

which completes the verification of the commutativity of the diagram above. By applying (9.4) to the classes modulo $I$ of constant functions, we obtain that for all $q \in M_{\kappa}$, there exists $\lambda_{q} \in R / J$ such that the set $Z_{q}=\left\{i \in \kappa^{+} \mid \dot{x}_{\lambda_{q}}(i) \neq q\right\}$ is finite; whence

$$
g_{i}\left(\left[\dot{x}_{\lambda_{q}}\right]_{I}\right)=q \cdot u_{i}, \text { for all } i \in \kappa^{+} \backslash\left(Y \cup Z_{q}\right) .
$$

Furthermore, there exists a subset $Z$ of $\kappa^{+}$containing $Y \cup \cup\left(Z_{q} \mid q \in M_{\kappa}\right)$, with at most $\kappa$ elements, such that $\dot{x}_{\lambda}$ is constant on $\kappa^{+} \backslash Z$, with value, say, $y_{\lambda} \in M_{\kappa}$, for all $\lambda \in R / J$. Hence,

$$
g_{i}\left(\left[\dot{x}_{\lambda}\right]_{I}\right)=\dot{x}_{\lambda} \wedge u_{i}=y_{\lambda} \cdot u_{i} \neq q_{\kappa} \cdot u_{i} \text {, for all }\langle\lambda, i\rangle \in(R / J) \times\left(\kappa^{+} \backslash Z\right) .
$$

Therefore, by (9.5) and (9.6), we obtain that

$$
\operatorname{im} g_{i}=\left(L_{\kappa}\left\lceil u_{i}\right) \backslash\left\{q_{\kappa} \cdot u_{i}\right\} \text {, for all } i \in \kappa^{+} \backslash Z\right. \text {. }
$$

Since both maps $\zeta$ and $\zeta_{i}$ are isomorphisms and the diagram above is commutative, the complement in $\boldsymbol{L}\left(a_{i} R\right)$ of the range of $\boldsymbol{L}\left(p_{i}\right)$ is also a singleton, for all $i \in \kappa^{+} \backslash Z$. Since $R / J \cong \mathrm{M}_{2}(E)$ and $a_{i} R \cong \mathrm{M}_{2}\left(E_{i}\right)$, we obtain, by Corollary 9.2 a contradiction.

Pushing the argument slightly further yields the following strong negative statement.

Theorem 9.4. There is no formula $\theta$ of $\mathcal{L}_{\infty, \infty}$ such that the class of 2-distributive coordinatizable lattices is the class of all models of $\theta$.

Proof. For any division ring $D$ with infinite cardinal $\kappa$, the ring of all almost constant $\kappa^{+}$-sequences of elements of $\mathrm{M}_{2}(D)$ coordinatizes the lattice $\bar{L}_{\kappa}$ defined in (9.1); whence $\bar{L}_{\kappa} \in \mathcal{C}$. We have seen in Theorem 9.3) that $L_{\kappa} \in \mathcal{N} \mathcal{C}$. Of course, $L_{\kappa}$ is a sublattice of $\bar{L}_{\kappa}$. Since $\kappa$ is arbitrarily large, it is sufficient, in order to conclude the proof, to establish that $L_{\kappa}$ is a $\mathcal{L}_{\kappa, \kappa}$-elementary submodel of $\bar{L}_{\kappa}$.

So we need to prove that $\bar{L}_{\kappa} \models \varphi$ implies that $L_{\kappa} \models \varphi$, for every $\langle\vee, \wedge\rangle$-sentence $\varphi$ in $\mathcal{L}_{\kappa, \kappa}$ with parameters from $L_{\kappa}$. The only nontrivial instance of the proof is to verify that $\bar{L}_{\kappa} \models \exists \overrightarrow{\mathrm{x}} \psi(\vec{a}, \overrightarrow{\mathrm{x}})$ implies that $L_{\kappa} \models \exists \overrightarrow{\mathrm{x}} \psi(\vec{a}, \overrightarrow{\mathrm{x}})$, for every formula $\psi$ in 
$\mathcal{L}_{\kappa, \kappa}$ for which we have already proved elementariness, with a list of parameters $\vec{a}=\left\langle a_{\xi} \mid \xi<\alpha\right\rangle$ from $L_{\kappa}$ and a list of free variables $\vec{x}=\left\langle x_{\eta} \mid \eta<\beta\right\rangle$, where $\alpha$, $\beta<\kappa$. So let us fix a list $\vec{b}=\left\langle b_{\eta} \mid \eta<\beta\right\rangle$ from $\bar{L}_{\kappa}$ such that $\bar{L}_{\kappa} \models \psi(\vec{a}, \vec{b})$. Since $\alpha$, $\beta<\kappa$, there are $\gamma<\kappa^{+}$and an automorphism $\sigma$ of $M_{\kappa+1}$ such that the following statements hold:

(i) $x(\zeta)=x(\infty)$, for all $x \in\left\{a_{\xi} \mid \xi<\alpha\right\} \cup\left\{b_{\eta} \mid \eta<\beta\right\}$ and all $\zeta \in \kappa^{+} \backslash \gamma$;

(ii) $\sigma(a)=a$, for all $a \in\left\{a_{\xi}(\infty) \mid \xi<\alpha\right\}$;

(iii) $\sigma(b) \in M_{\kappa}$, for all $b \in\left\{b_{\eta}(\infty) \mid \eta<\beta\right\}$.

Denote by $\tau$ the automorphism of $\bar{L}_{\kappa}$ defined by the rule

$$
\tau(x)(\zeta)=\left\{\begin{array}{ll}
x(\zeta), & \text { if } \zeta<\gamma, \\
\sigma(x(\zeta)), & \text { if } \gamma \leqslant \zeta,
\end{array} \quad \text { for all }\langle x, \zeta\rangle \in \bar{L}_{\kappa} \times \kappa^{+} .\right.
$$

Then $\tau$ fixes all $a_{\xi}$-s while the element $c_{\eta}=\tau\left(b_{\eta}\right)$ belongs to $L_{\kappa}$, for all $\eta<\beta$. From $\bar{L}_{\kappa} \models \psi(\vec{a}, \vec{b})$ it follows that $\bar{L}_{\kappa} \models \psi(\vec{a}, \vec{c})$, thus, by the induction hypothesis, $L_{\kappa} \models \psi(\vec{a}, \vec{c})$, and therefore $L_{\kappa} \models \exists \vec{x} \psi(\vec{a}, \vec{x})$.

\section{Appendix: Large partial three-Frames ARE Finitely AXIOMATizable}

For a positive integer $n$ and a bounded lattice $L$, we say that $L$ has a large partial $n$-frame, if there exists a homogeneous sequence $\left\langle a_{0}, \ldots, a_{n-1}\right\rangle$ of order $n$ in $L$ such that $L$ is generated by $a_{0}$ as a neutral ideal. It is clear that the existence of a large partial $(n+1)$-frame implies the existence of a large partial $n$-frame.

Having a large partial 3 -frame does not appear to be a first-order condition $a$ priori. However, we shall now prove that it is.

Proposition 10.1. Let $L$ be a complemented Arguesian lattice. Then $L$ has a large partial 3 -frame iff there are $a_{0}, a_{1}, a_{2}, b \in L$ such that

(i) $a_{0} \oplus a_{1} \oplus a_{2} \oplus b=1$;

(ii) $a_{i} \sim a_{j}$, for all distinct $i, j<3$;

(iii) $b \lesssim a_{0} \oplus a_{1}$.

In particular, for a complemented Arguesian lattice, having a large partial 3-frame can be expressed by a single first-order sentence.

Proof. It is obvious that the given condition implies that $\left\langle a_{0}, a_{1}, a_{2}\right\rangle$ is a homogeneous sequence such that the neutral ideal generated by $a_{0}$ is $L$.

Conversely, suppose that $L$ has a large partial 3-frame. We shall make use of the dimension monoid Dim $L$ of $L$ introduced in [27. As in [27, we denote by $\Delta(x, y)$ the element of Dim $L$ representing the abstract "distance" between elements $x$ and $y$ of $L$. Since $L$ has a zero, we put $\Delta(x)=\Delta(0, x)$, for all $x \in L$. We shall also use the result, proved in [27. Theorem 5.4], that the dimension monoid of a modular lattice is a refinement monoid.

Putting $\varepsilon=\Delta(1)$ and applying the unary function $\Delta$ to the parameters of a large partial 3-frame of $L$, we obtain that there are $n \in \mathbb{N}$ and $\alpha, \beta \in \operatorname{Dim} L$ such that the following relations hold:

$$
\begin{aligned}
3 \alpha+\beta & =\varepsilon ; \\
\beta & \leq n \alpha .
\end{aligned}
$$


Furthermore, by Jónsson's Theorem, $L$ is coordinatizable, thus normal as defined in [27. This implies easily the following statement:

$$
(\Delta(x)=\Delta(y) \text { and } x \wedge y=0) \Longrightarrow x \sim y \text {, for all } x, y \in L .
$$

Since $\operatorname{Dim} L$ is a refinement monoid, (10.2) implies (see [27] Lemma 3.1]) the existence of elements $\alpha_{k} \in \operatorname{Dim} L$, for $0 \leqslant k \leqslant n$, such that

$$
\alpha=\sum_{0 \leqslant k \leqslant n} \alpha_{k} \quad \text { and } \quad \beta=\sum_{0 \leqslant k \leqslant n} k \alpha_{k} .
$$

Put $\bar{\alpha}=\sum_{0 \leqslant k \leqslant n}\left(\left\lfloor\frac{k}{3}\right\rfloor+1\right) \alpha_{k}$ and $\bar{\beta}=\sum_{0 \leqslant k \leqslant n}\left(k-3\left\lfloor\frac{k}{3}\right\rfloor\right) \alpha_{k}$, where $\lfloor x\rfloor$ denotes the largest integer below $x$, for every rational number $x$. Hence we immediately obtain

$$
3 \bar{\alpha}+\bar{\beta}=\varepsilon .
$$

To prove that $\bar{\beta} \leq 2 \bar{\alpha}$, it suffices to prove that $k-3\left\lfloor\frac{k}{3}\right\rfloor \leqslant 2\left\lfloor\frac{k}{3}\right\rfloor+2$ for all $k \in$ $\{0,1, \ldots, n\}$, which is immediate.

Since the $\Delta$ function is a V-measure (see [27, Corollary 9.6]), there are $a_{0}, a_{1}$, $a_{2}, b \in L$ such that $a_{0} \oplus a_{1} \oplus a_{2} \oplus b=1$ in $L$ while $\Delta\left(a_{i}\right)=\bar{\alpha}$ for all $i<3$ and $\Delta(b)=\bar{\beta}$. Hence, by (10.3), $\left\langle a_{0}, a_{1}, a_{2}\right\rangle$ is a homogeneous sequence. Furthermore, $\Delta(b)=\bar{\beta} \leq 2 \bar{\alpha}=\Delta\left(a_{0} \oplus a_{1}\right)$, thus, by [27, Corollary 9.4], there are $x_{0} \leq a_{0}$, $x_{1} \leq a_{1}$, and $b_{0}, b_{1} \leq b$ such that $\Delta\left(x_{0}\right)=\Delta\left(b_{0}\right), \Delta\left(x_{1}\right)=\Delta\left(b_{1}\right)$, and $b=b_{0} \oplus b_{1}$. It follows again from (10.3) that $b=b_{0} \oplus b_{1} \sim x_{0} \oplus x_{1}$, whence $b \lesssim a_{0} \oplus a_{1}$.

We remind the reader of Jónsson's Extended Coordinatization Theorem (cf. Page 2), which states that for complemented Arguesian lattices, existence of a large partial 3-frame implies coordinatizability. In particular, lattices with a large partial 3-frame are not enough to settle Jónsson's Problem.

\section{OPEN PROBLEMS}

Some of our problems will be formulated in the language of descriptive set theory. We endow the powerset $\mathfrak{P}(X) \cong \mathbf{2}^{X}$ with the product topology of the discrete topological space $\mathbf{2}=\{0,1\}$, for any set $X$. So $\mathfrak{P}(X)$ is compact Hausdorff, metrizable in case $X$ is countable. Hence the space $\mathbf{S}=\mathfrak{P}\left(\omega^{2}\right) \times \mathfrak{P}\left(\omega^{3}\right) \times \mathfrak{P}\left(\omega^{3}\right)$, endowed with the product topology, is also compact metrizable. We endow it with its canonical recursive presentation (see Y.N. Moschovakis [23]).

We define $\mathbf{L}$ as the set of all triples $\xi=\langle E, M, J\rangle \in \mathbf{S}$ such that $E$ is a partial ordering on a nonzero initial segment $m$ of $\omega$ on which $J$ and $M$ are, respectively, the join and the meet operation with respect to $E$, and the lattice $L_{\xi}=\langle m, E, M, J\rangle$ is complemented modular.

Since stating that a structure is a complemented modular lattice can be expressed by a finite set of $\forall \exists$ axioms, it is not hard to verify that $\mathbf{L}$ is a $\Pi_{2}^{0}$ subset of $\mathbf{S}$. Put

$$
\mathbf{C L}=\left\{\xi \in \mathbf{L} \mid L_{\xi} \text { is coordinatizable }\right\},
$$

the set of real codes of coordinatizable lattices. As CL is defined by a second-order existential statement, it is a $\Sigma_{1}^{1}$ subset of $\mathbf{S}$.

Problem 1. Is CL a Borel subset of $\mathbf{S}$ ?

Problem 2. Is the set of real codes of countable complemented modular lattices admitting an orthocomplementation a Borel subset of $\mathbf{S}$ ? 
By using sheaf-theoretical methods, we could prove that the analogue of Problem 2 for 2-distributive lattices with spanning $M_{3}$ has a positive solution. In fact, the obtained condition is first-order.

Problem 3. Let $K$ be an elementary sublattice of a countable bounded lattice $L$. If $K$ is coordinatizable, is $L$ coordinatizable?

Our next problem is related to a possible weakening of the definition of coordinatizability.

Problem 4. If the join-semilattice $\mathrm{Sub}_{\mathrm{c}} M$ of all finitely generated submodules of a right module $M$ is a complemented lattice, is it coordinatizable?

Proposition 4.1 answers Problem 4 positively only in case $M$ is nœetherian.

Problem 5. Describe the elementary invariants of 2-distributive complemented modular lattices. Is the theory of 2-distributive complemented modular lattices decidable?

Problem 6. If a finite lattice $L$ can be embedded into some complemented modular lattice, can it be embedded into some finite complemented modular lattice?

A complemented modular lattice $L$ is uniquely coordinatizable, if there exists a unique (up to isomorphism) regular ring $R$ such that $L \cong \boldsymbol{L}(R)$.

Problem 7. Is the class of uniquely coordinatizable lattices a first-order class?

It is established in [15] that every complemented Arguesian lattice with a large partial 3-frame (see Section 10) is uniquely coordinatizable. The uniqueness part is [15], Theorem 9.4].

\section{ACKNOWLEDGMENT}

Part of this work was done during the author's visit at the TU Darmstadt in November 2002. The hospitality of the Arbeitsgruppe 14 and Christian Herrmann's so inspiring coaching on modular lattices are highly appreciated.

\section{REFERENCES}

[1] E. Artin, "Algèbre Géométrique", Cahiers Scientifiques, Fascicule 27, Gauthier-Villars, Bordas, Paris, 1978. x+212 p.

[2] M. K. Bennett, Lattices and Geometry, Lattice Theory and its Applications, K. A. Baker and R. Wille (eds.), Heldermann Verlag 1995, 27-50.

[3] G. Birkhoff, "Lattice Theory", Corrected reprint of the 1967 third edition. American Mathematical Society Colloquium Publications, Vol. 25. American Mathematical Society, Providence, R.I., 1979. vi+418 p.

[4] S. Burris and H. P. Sankappanavar, "A Course in Universal Algebra", The Millennium Edition, online manuscript available at http://www.thoralf.uwaterloo.ca, 1999. xvi+315 p. (Previously published as: Graduate Texts in Mathematics, 78. New York, Heidelberg, Berlin: Springer-Verlag. xvi+276 p., 1981. Out of print.)

[5] C.C. Chang and H. J. Keisler, "Model Theory", Studies in Logic and the Foundations of Mathematics 73, Elsevier Science B.V., North Holland, third edition, fourth impression, 1994. xvi+650 p.

[6] A. Day and D. Pickering, The coordinatization of Arguesian lattices, Trans. Amer. Math. Soc. 278 (1983), 507-522.

[7] K. D. Fryer and I. Halperin, The von Neumann coordinatization theorem for complemented modular lattices, Acta Sci. Math. (Szeged) 17 (1956), 203-249. 
[8] K. R. Goodearl, "Von Neumann Regular Rings", Second edition. Robert E. Krieger Publishing Co., Inc., Malabar, FL, 1991. xviii+412 p.

[9] G. Grätzer, "General Lattice Theory. Second edition", new appendices by the author with B. A. Davey, R. Freese, B. Ganter, M. Greferath, P. Jipsen, H. A. Priestley, H. Rose, E. T. Schmidt, S. E. Schmidt, F. Wehrung, and R. Wille. Birkhäuser Verlag, Basel, 1998. xx+663 p.

[10] M. Greferath and S. E. Schmidt, Projective Lattice Geometries, Appendix E in 9], 539-553.

[11] C. Herrmann, Alan Day's work on modular and Arguesian lattices, Algebra Universalis 34 (1995), 35-60.

[12] C. Herrmann, D. Pickering, and M. Roddy, A geometric description of modular lattices, Algebra Universalis 31, no. 3 (1994), 365-396.

[13] E. Hrushovski and B. Zilber, Zariski geometries, J. Amer. Math. Soc. 9, no. 1 (1996), 1-56.

[14] B. Jónsson, On the representations of lattices, Math. Scand. 1 (1953), 193-206.

[15] B. Jónsson, Representations of complemented modular lattices, Trans. Amer. Math. Soc. 97 (1960), 64-97.

[16] B. Jónsson, Extensions of von Neumann's Coordinatization Theorem, Proc. Symp. Pure Math. 2 (1961), 65-70.

[17] B. Jónsson, Representations of relatively complemented modular lattices, Trans. Amer. Math. Soc. 103 (1962), 272-303.

[18] B. Jónsson and G. Monk, Representation of primary Arguesian lattices, Pacific J. Math. 30 (1969), 95-130.

[19] R. N. McKenzie, G. F. McNulty, and W.F. Taylor, "Algebras, Lattices, Varieties. Volume I." The Wadsworth \& Brooks/Cole Mathematics Series. Monterey, California: Wadsworth \& Brooks/Cole Advanced Books \& Software, 1987. xii+361 p.

[20] F. Maeda, "Kontinuierliche Geometrien" (German), die Grundlehren der mathematischen Wissenschaften in Einzeldarstellungen mit besonderer Berücksichtigung der Anwendungsgebiete, Bd. 95. Springer-Verlag, Berlin-Göttingen-Heidelberg, 1958. x+244 p. (translated from Japanese by S. Crampe, G. Pickert, and R. Schauffler).

[21] F. Micol, "On representability of *-regular rings and modular ortholattices", PhD Thesis, Technische Universität Darmstadt, 2003.

[22] J. D. Monk and R. Bonnet, eds., "Handbook of Boolean Algebras", Volume 3, North-Holland, Amsterdam New-York Oxford Tokyo, xix+1367 p., 1989.

[23] Y. N. Moschovakis, "Descriptive Set Theory", Studies in Logic and the Foundations of Mathematics 100, North Holland Publishing Company, Amsterdam New-York Oxford, 1980. xii $+637 \mathrm{p}$.

[24] J. von Neumann, "Continuous Geometry", foreword by Israel Halperin. Princeton Mathematical Series, Vol. 25, Princeton University Press, Princeton, N. J. 1960. xi+299 p.

[25] M. Weese, Decidable Extensions of the Theory of Boolean Algebras, 985-1066 in 22.

[26] F. Wehrung, A uniform refinement property for congruence lattices, Proc. Amer. Math. Soc. 127, no. 2 (1999), 363-370.

[27] F. Wehrung, The dimension monoid of a lattice, Algebra Universalis 40, no. 3 (1998), 247411.

LMNO, CNRS UMr 6139, Université de Caen, Campus II, Département de Mathématiques, B.P. 5186, 14032 CAEN CEDEX, FRANCE

E-mail address: wehrung@math.unicaen.fr

URL: http://www.math.unicaen.fr/ ${ }^{\sim}$ wehrung 